\title{
CT scan and magnetic resonance diffusion-weighted imaging in the diagnosis and treatment of esophageal cancer
}

\author{
LEI GUO $^{1 *}$, LIULONG ZHANG ${ }^{2 *}$ and JIANSHE ZHAO ${ }^{3}$ \\ ${ }^{1}$ Department of MRI, Jinan Central Hospital, Jinan, Shandong 250013; ${ }^{2}$ Department of Radiology, \\ Dongying People's Hospital, Dongying, Shandong 257091; ${ }^{3}$ Department of Radiology, \\ Jinan Children's Hospital, Jinan, Shandong 250022, P.R. China
}

Received January 31, 2018; Accepted September 6, 2018

DOI: $10.3892 / 01.2018 .9532$

\begin{abstract}
Value of computed tomography (CT) scan and diffusion-weighted imaging (DWI) in the diagnosis and treatment of esophageal cancer was investigated. Seventy-eight patients with esophageal cancer treated in Jinan Central Hospital (Jinan, China) from January 2013 to June 2014 were selected. All patients underwent CT scan and DWI examination, and their clinical history data were analyzed. DWI was conducted. The short-term curative effect and the 3-year survival rate of patients in the high apparent diffusion coefficient (ADC) value group and the low ADC value group were compared; ADC values in the complete remission (CR) group and the partial remission (PR) group were compared. The difference in value between the length of esophageal lesions and the length of pathological specimens measured by CT scan was significantly different from that detected via DWI examination with $\mathrm{b}=600,800$ and $1,000 \mathrm{sec} / \mathrm{mm}^{2}$, respectively $(\mathrm{P}<0.05)$. The diagnostic rate of esophageal cancer via $\mathrm{CT}$ scan was significantly lower than that via DWI examination $(\mathrm{P}<0.05)$. After radiotherapy, the clinical control rate in the high ADC value group was significantly higher than that in the low ADC value group, and the 3-year survival rate in the former was significantly higher than that in the latter $(\mathrm{P}<0.05)$. In the 2nd week during radiotherapy and at the end of radiotherapy, the ADC values in the CR group were significantly higher than those in the PR group $(\mathrm{P}<0.05)$. In the 2 nd week during radiotherapy and at the end of radiotherapy, ADC values were used to predict the $\mathrm{CR}$ rate of radiotherapy for esophageal cancer, and the areas under the receiver operating characteristic (ROC) curve were 0.776 and 0.935 , respectively.
\end{abstract}

Correspondence to: Dr Jianshe Zhao, Department of Radiology, Jinan Children's Hospital, 23976 Jingshi Road, Jinan, Shandong 250022, P.R. China

E-mail: jqx6vy@163.com

${ }^{*}$ Contributed equally

Key words: computed tomography scan, diffusion-weighted imaging, esophageal cancer
Compared with CT scan, DWI has higher diagnostic rate and higher sensitivity. The length of esophageal tumor measured by DWI is close to that of pathological entity, which can guide the delineation of the target area of esophageal cancer.

\section{Introduction}

Esophageal cancer is a common malignant tumor in the digestive tract. The incidence rate differs between different ethnic groups and regions, and it is high in China and South Africa. With the changes in individuals' lifestyle and diet structure, the incidence rate of esophageal cancer has been increasing year by year around the world, and esophageal cancer has become one of the most serious diseases endangering human health (1). Dietary factors, especially the ingestion of large amounts of saturated fat and eating at high speed, are the main causes of esophageal cancer (2). The treatments for esophageal cancer include surgery, radiotherapy and chemotherapy. Although surgical resection is the only cure method, patients are definitely diagnosed with esophageal cancer in the middle and advanced stage due to the delay of the early diagnosis, relatively imperfect screening mechanisms and other factors, and they lose the best time for surgery. So the early diagnosis is particularly important (3). Computed tomography (CT) scan is used to evaluate curative effects and predict prognosis mainly from tumor size and morphological changes, but its accuracy is not high. Diffusion-weighted imaging (DWI) is used to evaluate curative effects from tumor molecular or cell level changes, and its apparent diffusion coefficient (ADC) can provide quantitative indicators for esophageal cancer before and after treatment, thus predicting the curative effect more accurately in an earlier stage $(4,5)$. In this study, the diagnosis and treatment of esophageal cancer were analyzed via CT scan and magnetic resonance imaging (MRI) scan for patients with esophageal cancer.

\section{Patients and methods}

General data. A total of 78 esophageal cancer patients admitted and treated in Jinan Central Hospital (Jinan, China) from January 2013 to June 2014 were randomly selected for retrospective analysis. Inclusion criteria: i) patients diagnosed with esophageal cancer by pathological examination; 
ii) patients receiving radiotherapy for the first time; iii) patients undergoing $\mathrm{CT}$ and MRI examinations with clear consciousness; and iv) patients who signed informed consent. Types of ulcer infiltration and stenosis were excluded. Patients with contraindications detected via MRI were excluded. The general data of patients are shown in Table I. This study was approved by the Ethics Committee of Jinan Central Hospital. Signed informed consents were obtained from the patients or the guardians.

Preparations before examinations. Patients fasted for $6 \mathrm{~h}$ prior to CT scan, and they were informed of the relevant precautions and received respiratory training (uniform, calm and slow breathing) to avoid poor image quality due to the change of respiratory rate. The patients were asked to have a light diet on the day prior to MRI examination so as to avoid MRI signal changes due to high-fat foods.

$C T$ examination. The patients were instructed to put their hands on both sides of the pillow, and they were placed in the supine position and scanned with a dual-source CT scanner (Siemens AG, Munich, Germany) (thickness, $3.0 \mathrm{~mm}$; layer distance, $3.0 \mathrm{~mm}$ ). The scanning sites were the chest and upper abdomen, ranging from the chest entrance down to the lower pole of the kidney. The scanned images were transmitted to the workstation. Two senior imaging physicians applied Syntegra software (Philips Medical Systems, Andover, MA, USA) for image fusion, target area delineation and lesion length calculation based on spinal imaging, skeletal structure and localized $\mathrm{CT}$ images.

MRI examination. The patients were guided to lie down in the supine position and scanned by conventional plain MRI [T1-weighted imaging (T1WI) and T2WI] and DWI using the Avanto 1.5T magnetic resonance scanner (Siemens AG). The scanning sites were the chest and upper abdomen: i) T1WI axial scan [repetition time (TR), $140 \mathrm{msec}$; time to echo (TE), 2.5 msec; matrix, 256x 256; field of view (FOV) squared, $36 \times 36 \mathrm{~cm}$; flip angle, $70^{\circ}$; bandwidth (BW), $280 \mathrm{~Hz}$; layer thickness, $6.0 \mathrm{~mm}$; and interlayer interval, 20\%) for $2 \mathrm{~min}$ and $15 \mathrm{sec}$. ii) T2WI axial scan (TR, 1,580 msec; TE, $72 \mathrm{msec}$; matrix, 384x276; FOV, 35x35 cm; flip angle, 140 ; BW, 315; layer thickness, $6.0 \mathrm{~mm}$; and interlayer interval, $20 \%$ ) for $1 \mathrm{~min}$ and $30 \mathrm{sec}$. iii) DWI axial scan (TR, 6,800 msec; TE, 70 msec; matrix, 128x128; FOV, 40x40 cm; BW, 260; layer thickness, $4.0 \mathrm{~mm}$; and interlayer interval, $0 \%$ ) for $9 \mathrm{~min}$ and $30 \mathrm{sec}$ using the single-shot spin-echo-echo-planar imaging (SE-EPI) sequence. Four different diffusion-sensitive factors were simultaneously collected during the scans: $b=0$, 600,800 , and $1,000 \mathrm{sec} / \mathrm{mm}^{2}$. The DWI images were fused to obtain the corresponding ADC images, and the lesion lengths at different $b$ values were calculated. The target area was delineated with $b=600 \mathrm{sec} / \mathrm{mm}^{2}$. Patients were divided into high $\mathrm{ABC}\left(>1.5 \times 10^{-3} \mathrm{~mm}^{2} / \mathrm{sec}\right)$ group and low ADC $\left(\leq 1.5 \times 10^{-3} \mathrm{~mm}^{2} / \mathrm{sec}\right)$ group. The length of the lesion was selected for providing a more accurate location for surgical treatment, to improve the effectiveness of surgery, to effectively predict the prognosis and to make reasonable operation plan and postoperative radiotherapy and chemotherapy. Before a pathological examination, the length of the lesion can be
Table I. General data of study subjects.

Item

Tested subjects $(\mathrm{n}=78)$

Average age (years)

$55.78 \pm 6.54$

Sex (male/female)

$53 / 25$

Tumor site [n (\%)]

Neck

Upper thoracic part

Middle and lower thoracic part

$59(75.64)$

Pathological type [n (\%)]

Squamous cell carcinoma

Adenocarcinoma

Small cell carcinoma

$7(8.97)$

obtained by imaging examination, which has certain guiding value for the diagnosis and treatment of esophageal cancer.

\section{Indicator evaluation}

Evaluation criteria for short-term curative effects. i) Complete remission (CR): the tumor completely disappears, no stenosis or slight stenosis occurs in the esophageal lumen, and mucosa basically returns to normal; ii) partial remission (PR): partial esophageal lesions disappear, but obvious stenosis is found in the lumen; and iii) no remission (NR): at the end of radiotherapy, the lesion is not improved or aggregated, and there are still residual lesions. Clinical control rate $=(\mathrm{CR}+\mathrm{PR}) /$ the total number of cases x100\% (1).

$A D C$ value calculation. DWI images with $\mathrm{b}=0,600$, 800 , and $1,000 \mathrm{sec} / \mathrm{mm}^{2}$ were selected and fused to obtain the corresponding ADC images. The manifested largest and most clear sections of esophageal lesions were selected as the region of interest (ROI), and the ROI of each ADC image should be selected in the same area. Each ROI was measured 3 times, and the average value was taken as the final ADC value of each ROI (6).

Statistical methods. Statistical Product and Service Solutions (SPSS) 19.0 analysis software (IBM Corp., Armonk, NY, USA) was used. Measurement data were expressed as mean \pm standard deviation, and detected by t-test. Count data were expressed as percentage, and detected by $\chi^{2}$ test. ANOVA was used for comparison of multiple groups and Dunnett's test was the post hoc test. Receiver operating characteristic (ROC) curve analyses were conducted for diagnostic methods (CT and MRI) and efficacy prediction. Survival curves were drawn using the Kaplan-Meier method, and the long-rank test was employed for survival analysis. Test significance level $\alpha=0.05$.

\section{Results}

Comparison of the length of esophageal lesions measured by different examination methods. The difference in value between the length of esophageal lesions and the length of pathological specimens measured by CT scan was significantly different from that detected via DWI examination with $b=600$, 
Table II. Comparison of the length of esophageal lesions measured by different examination methods $(\mathrm{cm})$.

\begin{tabular}{lcc}
\hline Method & Length & $\begin{array}{c}\text { Difference } \\
\text { value }\end{array}$ \\
\hline $\begin{array}{l}\text { Pathological entity } \\
\text { measurement }\end{array}$ & $4.56 \pm 1.43$ & \\
CT scan & $5.67 \pm 1.65$ & $1.15 \pm 0.59$ \\
$\begin{array}{l}\text { DWI examination } \\
\text { b=600 sec } / \mathrm{mm}^{2}\end{array}$ & $4.41 \pm 1.62$ & $0.19 \pm 0.36^{\mathrm{a}}$ \\
$\mathrm{b}=800 \mathrm{sec} / \mathrm{mm}^{2}$ & $3.97 \pm 1.45$ & $0.65 \pm 0.42^{\mathrm{a}, \mathrm{b}}$ \\
$\mathrm{b}=1,000 \mathrm{sec} / \mathrm{mm}^{2}$ & $3.84 \pm 1.62$ & $0.84 \pm 0.53^{\mathrm{a}, \mathrm{b}, \mathrm{c}}$
\end{tabular}

${ }^{\mathrm{a} P}<0.05$ compared with $\mathrm{CT}$ scan, ${ }^{\mathrm{b}} \mathrm{P}<0.05$ compared with $\mathrm{b}=600 \mathrm{sec} / \mathrm{mm}^{2}$, and ${ }^{\mathrm{c}} \mathrm{P}<0.05$ compared with $\mathrm{b}=800 \mathrm{sec} / \mathrm{mm}^{2}$. CT, computed tomography; DWI, diffusion-weighted imaging.

Table III. Comparison of the diagnostic rate of esophageal cancer by different imaging examination methods [n (\%)].

\begin{tabular}{lcc}
\hline Method & $\mathrm{n}$ & Diagnostic rate \\
\hline CT scan & 78 & $69(88.46)$ \\
DWI examination & 78 & $77(98.72)$ \\
$\chi^{2}$ & & 5.236 \\
P-value & & 0.022 \\
\hline
\end{tabular}

CT, computed tomography; DWI, diffusion-weighted imaging.

800 and $1,000 \mathrm{sec} / \mathrm{mm}^{2}$, respectively $(\mathrm{P}<0.05)$, and the feasibility of DWI with $b=600 \mathrm{sec} / \mathrm{mm}^{2}$ was the highest (Table II).

Comparison of the diagnostic rate of esophageal cancer by different imaging examination methods. The diagnostic rate of esophageal cancer via CT scan was obviously lower than that via DWI examination $(\mathrm{P}<0.05)$. The area under the ROC curve of diagnostic rate of esophageal cancer by CT scan was 0.794 , with sensitivity of $70.3 \%$ and specificity of $69.4 \%$, while that by DWI was 0.845 , with sensitivity of $87.6 \%$ and specificity of $82.5 \%$ (Table III and Fig. 1).

Comparison of the curative effects in the two groups of patients. After radiotherapy, the 1-year clinical control rate in the high ADC value group before radiotherapy was significantly higher than that in the low ADC value group, and the 3 -year survival rate in the former was significantly higher than that in the latter (Table IV and Fig. 2).

Comparison of the ADC value of primary esophageal foci of patients in the CR and the PR groups in different time-points. There was no significant difference in the ADC value of primary esophageal foci before radiotherapy between the CR and the $\mathrm{PR}$ group $(\mathrm{P}>0.05)$. At the 2 nd week during radiotherapy and at the end of radiotherapy, ADC values in the $\mathrm{CR}$ group were significantly higher than those in the PR group
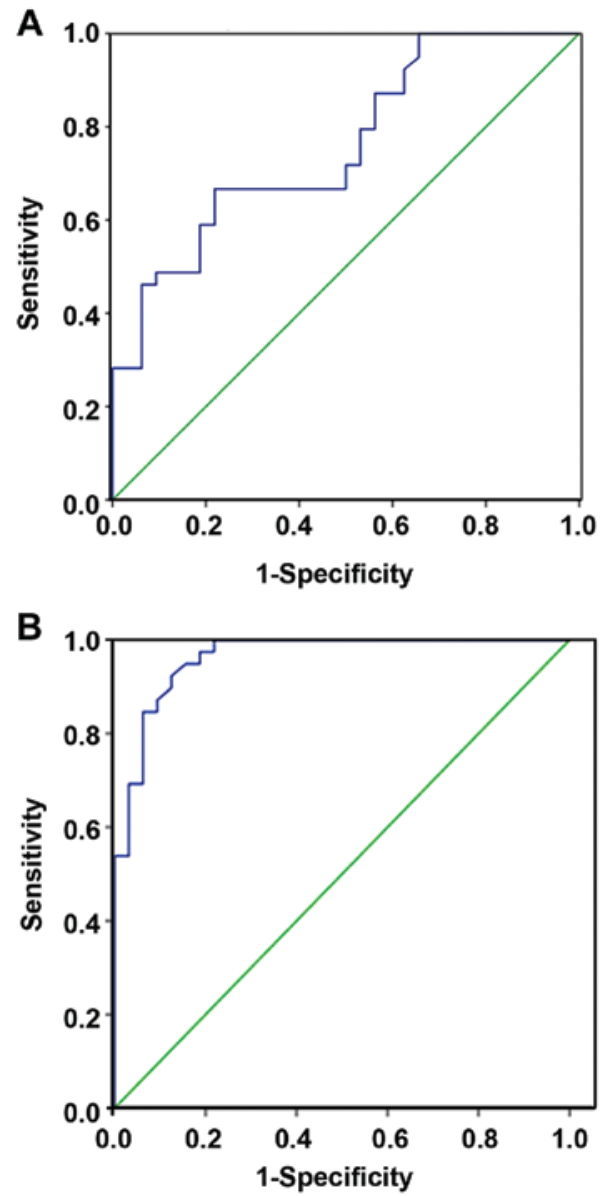

Figure 1. Comparison of the length of esophageal lesions measured by different examination methods. ROC curve of (A) CT scan and (B) DWI examination. The ROC curve showed that the area under the ROC curve of the diagnostic rate of esophageal cancer by CT scan was 0.794 , the sensitivity was $70.3 \%$ and the specificity was $69.4 \%$. The ROC curve showed that the area under the ROC curve of the diagnostic rate of esophageal cancer by DWI examination was 0.845 , the sensitivity was $87.6 \%$ and the specificity was $82.5 \%$. The area of CT scan was smaller than that of DWI examination $(\mathrm{P}<0.05)$. The sensitivity and specificity of $\mathrm{CT}$ scan were lower than that of DWI examination $(\mathrm{P}<0.05)$. ROC, receiver operating characteristic; $\mathrm{CT}$, computed tomography; DWI, diffusion-weighted imaging.

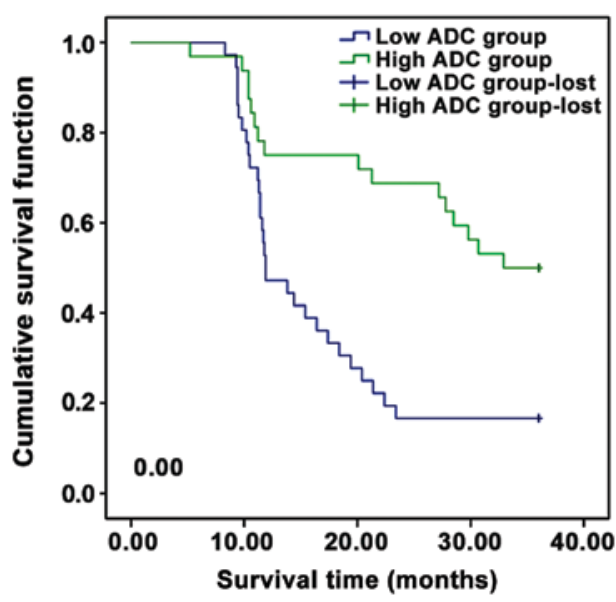

Figure 2. Survival curves of patients of the two groups with different ADC values. Survival function analysis showed that the survival function of the low ADC value group was decreased faster than that of the high ADC value group. The survival function of the low ADC value group was $\sim 0$ at $<25$ months, while that of the high ADC value group was $\sim 0$ at $<35$ months. ADC, apparent diffusion coefficient. 
Table IV. Comparison of the curative effects in the two groups of patients [n (\%)].

\begin{tabular}{lccccc}
\hline Group & $\mathrm{n}$ & $\mathrm{CR}$ & $\mathrm{PR}$ & Clinical control rate & 3-year survival rate \\
\hline High ADC value group & 41 & $21(51.22)$ & $16(39.02)$ & $37(90.24)$ & $24(58.54)$ \\
Low ADC value group & 37 & $11(29.73)$ & $14(37.84)$ & $25(67.57)$ & $12(32.43)$ \\
$\chi^{2}$ & & & & 4.822 & 4.334 \\
P-value & & & & 0.028 & 0.037
\end{tabular}

CR, complete remission; PR, partial remission; ADC, apparent diffusion coefficient.

Table V. Comparison of the ADC values of primary esophageal foci at different time-points of patients of the CR and the PR groups $\left(10^{-3} \mathrm{~mm}^{2} / \mathrm{sec}\right)$.

\begin{tabular}{|c|c|c|c|c|c|c|}
\hline Group & $\mathrm{n}$ & $\begin{array}{l}\text { Before } \\
\text { radiotherapy }\end{array}$ & $\begin{array}{l}\text { At } 2 \text { weeks after } \\
\text { radiotherapy }\end{array}$ & $\begin{array}{l}\text { At the end of } \\
\text { radiotherapy }\end{array}$ & $\mathrm{F}$ & P-value \\
\hline $\mathrm{CR}$ group & 32 & $1.63 \pm 0.65$ & $2.23 \pm 0.53$ & $2.84 \pm 0.56$ & 52.163 & $<0.001$ \\
\hline PR group & 30 & $1.46 \pm 0.57$ & $1.68 \pm 0.48$ & $1.96 \pm 0.47$ & 48.826 & $<0.001$ \\
\hline $\mathrm{t}$ & & 1.092 & 4.273 & 6.679 & & \\
\hline P-value & & 0.279 & $<0.001$ & $<0.001$ & & \\
\hline
\end{tabular}

ADC, apparent diffusion coefficient; CR, complete remission; PR, partial remission.

A

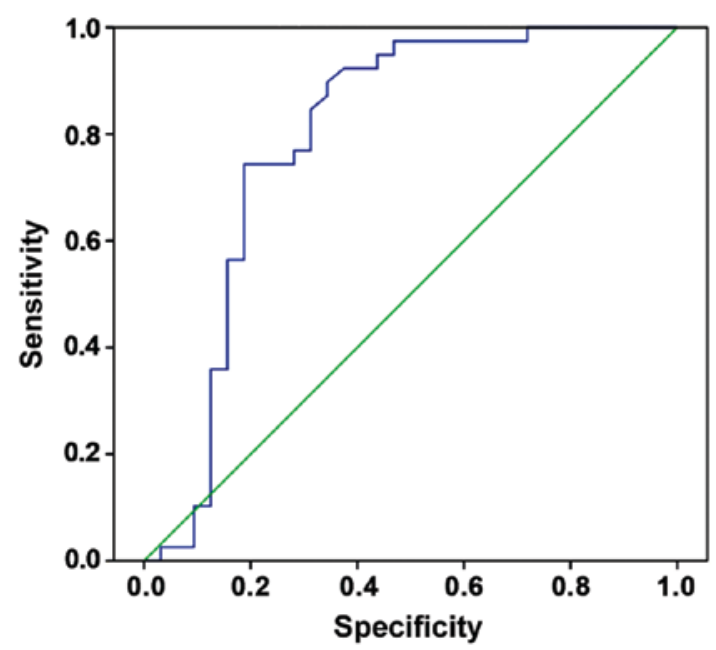

B

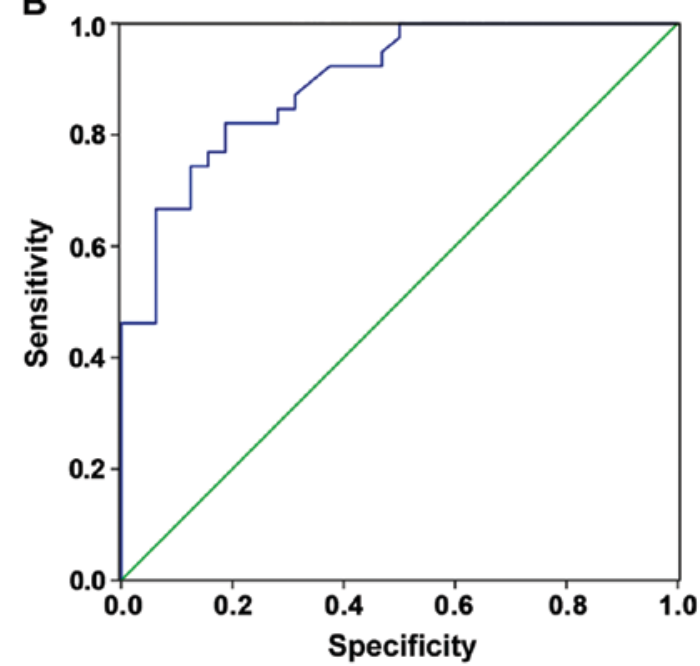

Figure 3. Comparison of the ADC value of primary esophageal foci of patients of the CR and the PR groups in different time-points. (A) ROC curve of ADC value at the 2nd week during radiotherapy; (B) ROC curve of ADC value at the end of radiotherapy. The ROC curve showed that the area of the group at the end of radiotherapy (0.935) was bigger than that of the group at the 2 nd week during radiotherapy $(0.776)$, the critical value at the end of radiotherapy $\left(1.46 \times 10^{-3} \mathrm{~mm}^{2} / \mathrm{sec}\right)$ was higher than that at the 2 nd week during radiotherapy $\left(1.38 \times 10^{-3} \mathrm{~mm}^{2} / \mathrm{sec}\right)$, the sensitivity rate at the end of radiotherapy $(91.5 \%)$ was higher than that at the 2nd week during radiotherapy (62.3\%), and the specificity at the end of radiotherapy (83.7\%) was lower than that at the 2 nd week during radiotherapy (98.3\%). ADC, apparent diffusion coefficient; CR, complete remission; PR, partial remission; ROC, receiver operating characteristic.

$(\mathrm{P}<0.05)$. At the 2nd week during radiotherapy and at the end of radiotherapy, ADC values were used to predict the $\mathrm{CR}$ rate of radiotherapy for esophageal cancer, and the areas under the ROC curve were 0.776 and 0.935 , respectively, with critical values of $1.38 \times 10^{-3}$ and $1.46 \times 10^{-3} \mathrm{~mm}^{2} / \mathrm{sec}$, sensitivities of 62.3 and $91.5 \%$, and specificities of $98.3 \%$ and $83.7 \%$ (Table $\mathrm{V}$ and Fig. 3).

\section{Discussion}

Esophageal cancer is a malignant tumor occurring in esophageal epithelial tissues, which is caused by abnormal cell proliferation. The invasion ability of esophageal cancer is strong, and it is mostly located in the middle part of the chest, but less distributed in the upper and lower parts of the 
chest. The majority are esophageal squamous cell carcinoma, but adenocarcinoma and small cell carcinoma are rare (7). Esophageal cancer is a malignant tumor that occurs in esophageal epithelial tissues. Its clinical stage can be divided into stage I, II and III. Esophageal cancer occurs frequently at the age of $>40$ years, with higher frequency in men than women, and its incidence and mortality rates rank 4th among malignant tumors (8). Esophageal cancer is induced by many factors, including excessively high eating speed, ingestion of too hot foods, foods with mycotoxins or large amounts of nitrosamines, smoking, excessive drinking, poor oral hygiene, nutritional deficiencies, human papillomavirus infections and genetic factors (9). Patients are often diagnosed with esophageal cancer in the middle and advanced stage with poor prognosis and low survival rate, so improving the early screening and diagnosis of esophageal cancer has important clinical significance (10).

Endoscopic examination is usually used in clinical diagnosis of esophageal carcinoma, but it is invasive and patient compliance is poor, so other imaging techniques, such as CT and MRI, are often used. CT scan has the advantages of high scanning speed, clear images and little susceptibility to peripheral organs. With the rapid development of medical imaging technology, CT has been developed from 4 rows in the past to 64 rows and even more, thus resulting in shorter scanning time, higher resolution and less motion artifact interference. Therefore, CT scan has become a main clinical diagnostic method for many diseases (11). MRI is a new type of medical imaging technology developed on the basis of magnetic resonance display. It has the advantages of no radiation damage, multi-orientation and multi-sequence imaging and high spatial resolution, and it is widely used in the examination of various diseases (12). The results of this study showed that the diagnostic rate of esophageal cancer by $\mathrm{CT}$ scan was significantly lower than that by DWI examination. The difference in value between the length of esophageal lesions and the length of pathological specimens measured by CT scan was significantly different from that detected via DWI examination with $\mathrm{b}=600,800$ and $1,000 \mathrm{sec} / \mathrm{mm}^{2}$, respectively $(\mathrm{P}<0.05)$. This is because CT scan examines the morphological changes in tumors, and there are deficiencies in the determination of the esophageal lesion range and lymph node metastasis of esophageal lesions. The length of esophageal lesions measured by CT scan is too large, since tumor necrosis occurs followed by secondary infection, so that edema occurs in normal esophageal tissues, and CT scan cannot be effectively performed for differential diagnosis. When esophageal cancer is in stage III, lymph node metastasis and esophageal wall adhesion occur, so it is difficult for CT scan to distinguish, which is also influenced by the partial volume effect of $\mathrm{CT}$, resulting in the poor accuracy of the doctor outlining the target area, and thus affecting the curative effect of radiotherapy (13). DWI, as a new MRI technique, utilizing the dispersed phase effect caused by a special magnetic resonance sequence to reflect the microscopic diffusion motion of water molecules in tissues at the macro-imaging, so as to form DWI images, which can reflect the function state of the exchange of water molecules in diseased tissues. DWI can distinguish lymph nodes and blood vessels without enhancers, so the length of esophageal lesions measured by DWI is relatively accurate. Therefore, the sensitivity and specificity of the diagnosis of esophageal cancer by DWI are significantly higher than those by CT scan, thus providing important information for delineating the target area $(5,14)$. $b$ is one of the important parameters of DWI technique. The quality of DWI images and the authenticity of water molecule diffusion are directly related to $b$. The smaller the $b$ is, the lower the degree of diffusion, the smaller the influence on signals and the higher the image quality will be. Therefore, DWI has the highest credibility when $b=600 \mathrm{sec} / \mathrm{mm}^{2}$, which can be used to draw the target area (15).

ADC value is one of the quantitative indicators of DWI, which can quantitatively evaluate the diffusion state of water molecules in the tissue structure (16). The study results revealed that the 1-year clinical control rate in the high ADC value group before radiotherapy was significantly higher than that in the low ADC value group, and the 3-year survival rate in the former was significantly higher than that in the latter. There was no significant difference in the ADC value of esophageal primary foci between the CR and PR group $(\mathrm{P}>0.05)$. At the 2 nd week during radiotherapy and at the end of radiotherapy, the ADC values in the CR group were significantly higher than those in the PR group $(\mathrm{P}<0.05)$. This is because the more active the proliferation of tumor cells in esophageal cancer is, the larger the number of tumor cells and the closer the arrangement are, which leads to the smaller extracellular space and seriously restricted diffusion of water molecules, and the lower the ADC value will be (17). In addition, ADC value can reflect the degree of tumor load. With the progression of treatment, esophageal lesions are shrinking; tumor cells continue to apoptosis, and thus the number of cells and density are decreased, the extracellular space is increased; the limitation of diffusion of water molecules is alleviated, so ADC value is elevated (18). Changes in the ADC value during and after treatment reflect the different sensitivities of tumor tissues to treatment responses. The more obvious the curative effect is, the higher the ADC value, the better the prognosis of patients, and the higher the survival rate will be (19).

In summary, compared with CT scan, DWI examination is more objective and accurate in the diagnosis of esophageal cancer, and DWI is of great significance in predicting the curative effect of radiotherapy on esophageal cancer. ADC value can be used as a quantitative index to predict the sensitivity of esophageal cancer to radiotherapy. Due to the small sample size and short follow-up period, there is still a need to further expand the sample size and conduct long-term research in the future.

\section{Acknowledgements}

Not applicable.

Funding

No funding was received.

\section{Availability of data and materials}

The datasets used and/or analyzed during the current study are available from the corresponding author on reasonable request. 


\section{Authors' contributions}

LG collected and analyzed the general data of the patients. LZ assisted with ADC value calculation. LG and JZ were responsible for interpreting the scan results. All authors read and approved the final manuscript.

\section{Ethics approval and consent to participate}

This study was approved by the Ethics Committee of Jinan Central Hospital (Jinan, China). Signed informed consents were obtained from the patients or the guardians.

\section{Patient consent for publication}

Not applicable.

\section{Competing interests}

The authors declare that they have no competing interests.

\section{References}

1. Probst A, Aust D, Märkl B, Anthuber M and Messmann H: Early esophageal cancer in Europe: Endoscopic treatment by endoscopic submucosal dissection. Endoscopy 47: 113-121,2015.

2. Oyama T, Hotta K and Tomori A: Endoscopic submucosal dissection for superficial esophageal squamous cell carcinoma and adenocarcinoma. Gastrointest Endosc 65: AB110, 2007.

3. van der Sluis PC, Ruurda JP, Verhage RJ, van der Horst S, Haverkamp L, Siersema PD, Borel Rinkes IH, Ten Kate FJ and van Hillegersberg R: Oncologic long-term results of robot-assisted minimally invasive thoraco-laparoscopic esophagectomy with two-field lymphadenectomy for esophageal cancer. Ann Surg Oncol 22 (Suppl 3): S1350-S1356, 2015.

4. Drummond MB, Lambert AA, Hussien AF, Lin CT, Merlo CA, Wise RA, Kirk GD and Brown RH: HIV infection is independently associated with increased CT scan lung density. Acad Radiol 24: 137-145, 2017.

5. Boone D, Taylor SA and Halligan S: Diffusion weighted MRI: Overview and implications for rectal cancer management. Colorectal Dis 15: 655-661, 2013.

6. Nozaki I, Hato S, Hori S, Nishide N and Kurita A: Incidence of food residue interfering with postoperative endoscopic examination for gastric pull-up after esophagectomy. Esophagus 13: 195-199, 2016.

7. Tachibana M, Kinugasa S, Hirahara N and Yoshimura H: Lymph node classification of esophageal squamous cell carcinoma and adenocarcinoma. Eur J Cardiothorac Surg 34: 427-431, 2008.
8. Domper Arnal MJ, Ferrández Arenas Á and Lanas Arbeloa Á: Esophageal cancer: Risk factors, screening and endoscopic treatment in Western and Eastern countries. World J Gastroenterol 21: 7933-7943, 2015.

9. Xu W, Liu Z, Bao Q and Qian Z: Viruses, other pathogenic microorganisms and esophageal cancer. Gastrointest Tumors 2: 2-13, 2015.

10. Lin G, Han SY, Xu YP and Mao WM: Increasing the interval between neoadjuvant chemoradiotherapy and surgery in esophageal cancer: A meta-analysis of published studies. Dis Esophagus 29: 1107-1114, 2016.

11. Chou CT, Chen RC, Lin WC, Ko CJ, Chen CB and Chen YL: Prediction of microvascular invasion of hepatocellular carcinoma: Preoperative CT and histopathologic correlation. AJR Am J Roentgenol 203: W253-9, 2014.

12. Ganten MK, Schuessler M, Bäuerle T, Muenter M, Schlemmer HP, Jensen A, Brand K, Dueck M, Dinkel J, Kopp-Schneider A, et al: The role of perfusion effects in monitoring of chemoradiotherapy of rectal carcinoma using diffusion-weighted imaging. Cancer Imaging 13: 548-556, 2013.

13. Tchelebi L and Ashamalla H: Overcoming the hurdles of using $\mathrm{PET} / \mathrm{CT}$ for target volume delineation in curative intent radiotherapy of non-small cell lung cancer. Ann Transl Med 3: 191, 2015.

14. Anderson SW, Barry B, Soto JA, Ozonoff A, O'Brien M and Jara H: Quantifying hepatic fibrosis using a biexponential model of diffusion weighted imaging in ex vivo liver specimens. Magn Reson Imaging 30: 1475-1482, 2012.

15. GigantiF, Salerno A,Ambrosi A,ChiariD, Orsenigo E, Esposito A, Albarello L, Mazza E, Staudacher C, Del Maschio A, et al: Prognostic utility of diffusion-weighted MRI in oesophageal cancer: Is apparent diffusion coefficient a potential marker of tumour aggressiveness? Radiol Med (Torino) 121: 173-180, 2016.

16. Purushotham A, Campbell BC, Straka M, Mlynash M, Olivot JM, Bammer R, Kemp SM, Albers GW and Lansberg MG: Apparent diffusion coefficient threshold for delineation of ischemic core. Int J Stroke 10: 348-353, 2015.

17. Mori N, Ota H, Mugikura S, Takasawa C, Ishida T, Watanabe G, Tada H, Watanabe M, Takase K and Takahashi S: Luminal-type breast cancer: Correlation of apparent diffusion coefficients with the Ki-67 labeling index. Radiology 274: 66-73, 2015.

18. PLOS ONE Staff: Correction: Correlation of the apparent diffusion coefficient (ADC) with the standardized uptake value (SUV) in lymph node metastases of non-small cell lung cancer (NSCLC) patients using hybrid 18F-FDG PET/MRI. PLoS One 10: e0120606, 2015.

19. Boesen L, Chabanova E, Løgager V, Balslev I and Thomsen HS: Apparent diffusion coefficient ratio correlates significantly with prostate cancer gleason score at final pathology. J Magn Reson Imaging 42: 446-453, 2015.

This work is licensed under a Creative Commons

Attribution-NonCommercial-NoDerivatives 4.0 International (CC BY-NC-ND 4.0) License. 